\title{
Recent Results From the MINERvA Experiment
}

\author{
Edgar Valencia-Rodriguez ${ }^{* \dagger}$ \\ William \& Mary \\ E-mail: evalenciarodri@wm.edu
}

\begin{abstract}
MINERvA is a dedicated neutrino-nucleus scattering experiment with multiple nuclear targets in the Neutrinos from the Main Injector (NuMI) beam line at Fermilab. MINERvA is providing high precision measurements of neutrino interaction cross sections in the 1 to $20 \mathrm{GeV}$ energy range in support to current and future oscillation experiments. MINERvA can also provide information about the structure of protons and neutrons and about the dynamics affecting neutrino-nucleus interactions. The heart of the MINERvA detector is a fine-grained scintillating tracking chamber, surrounded by electromagnetic and hadronic calorimeters. Carbon, iron and lead targets are used to study the effect of the nuclear medium on neutrino-induced interactions. This document summarizes the most recent MINERvA results.
\end{abstract}

The 19th International Workshop on Neutrinos from Accelerators-NUFACT2017

25-30 September, 2017

Uppsala University, Uppsala, Sweden

\footnotetext{
* Speaker.

${ }^{\dagger}$ On Behalf of the MINERvA Collaboration
} 


\section{Introduction}

Neutrino interaction cross section is one of the main sources of systematics in neutrino oscillation experiments. Uncertainties on the cross section measurements come mostly from the models used by the neutrino events generator. High precision data measurements, like MINERvA's, are needed to improve these models. Some current and future neutrino oscillation experiments operate in the 1 to $20 \mathrm{GeV}$ range where several processes happen. Quasi-elastic, resonance and deep inelastic scattering can be signal or background in oscillation experiments and the MINERvA Experiment can greatly help with the characterization of these processes.

\section{Flux Predictions}

Geant4 simulation of the beamline and hadroproduction data from NA49 and NA61 experiments are used to predict the neutrino flux. Figure 1 shows the current MINERvA flux prediction for the NuMI Low Energy mode ${ }^{1}$. The same technique can be used to predict the DUNE flux that shall have similar hadron production uncertainties. The Medium Energy data set shall have errors in the order of 5\% once constraints of in situ measurements are added. Sources of the focusing uncertainties in Low Energy beam configuration come, among others, from the baffle scraping, POT counting, horn current and water layer as shown in Figure 1.

\section{Normalization from Neutrino Electron Scattering}

MINERvA uses the very well known electroweek neutrino electron scattering process ${ }^{2}$ to constrain the flux. Cuts in the energy deposited at the beginning of the shower, in the electron energy and in the electron angle are applied to isolate this process. Figure 2 shows the electron energy spectrum. These statistically limited results reduce the MINERvA flux uncertainties as a function of the energy by 10 to $20 \%$.

\section{Flux Shape from Low-Nu Scattering}

Low hadronic recoil energy charged current scattering with the Low-Nu method [3] confirms the flux prediction made with external data. At lower energies the shape of the flux is determined by the low-nu events where the majority of the energy goes into the final state muon. Muon energy reconstruction is then the main contributor to uncertainties. This is the first time that low-nu based technique was used in the NuMI beam line. This measurement is the lowest energy application of low-nu flux technique and is also applicable to future neutrino beam at few $\mathrm{GeV}$.

\section{Inclusive Charged Current Scattering}

Future long-baseline oscillation experiments will use neutrino and antineutrino beams below $10 \mathrm{GeV}$ to measure oscillation parameters and $\mathrm{CP}$ violation phase. MINERvA has the most precise

\footnotetext{
${ }^{1}$ Comparing to our old predictions the new result has improvements in the beamline geometry and updates in the focusing uncertainties, hadronic interactions and beamline absorptions.

${ }^{2} \mathrm{~A}$ very forward electron in the final state is the main signature of this kind of event.
} 

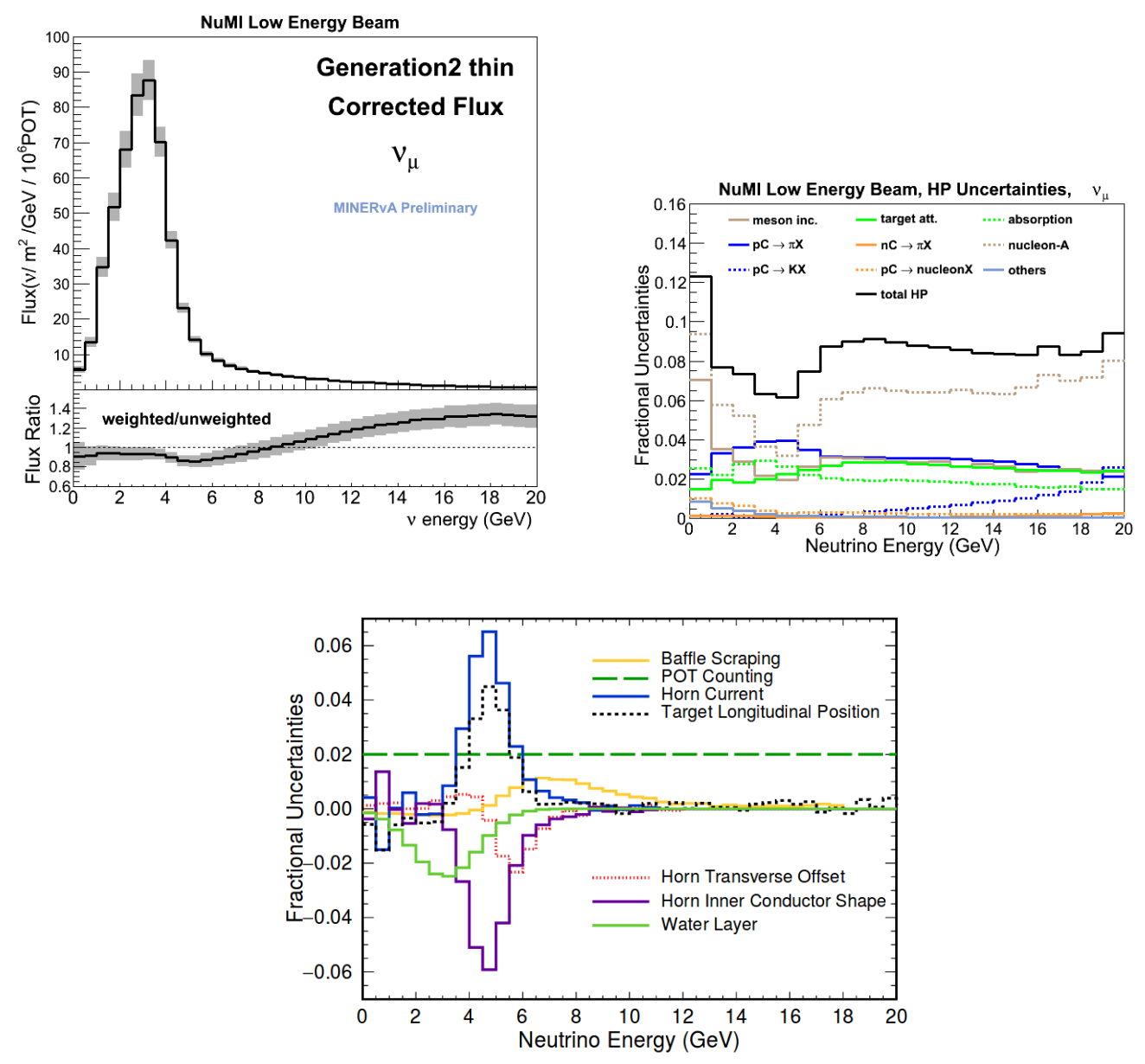

Figure 1: Top left: Low energy neutrino flux prediction; Top right: Hadron Production uncertainties; Bottom: Neutrino flux uncertainties. [1]

(below 5\% ) antineutrino cross section result below $6 \mathrm{Gev}$, where errors are dominated by the statistical precision. Figure 4 shows the cross section for inclusive charged current neutrino and antineutrino scattering. MINERvA results are in agreement with those of NOMAD and MINOS but extend to lower energies. Studies of CP asymmetry are sensitive to the antineutrino neutrino coss section ratio. The ratio between neutrino and antineutrino cross sections in Figure 4 agrees with previous results. Common systematics, like the normalization cross section model used, for neutrino and antineutrino are partiality canceled in the ratio. The main uncertainty is statistical.

\section{Neutrino and Antineutrino Single Pions Production}

Neutrino and antineutrino pion production cross sections are important for the understanding of nuclear effects. MINERvA selects neutrino and antineutrino samples by separating CC pion events in two semi inclusive channels: one for single pion resonant production for neutrinos and the other for single neutral pion production for antineutrinos, that could be generated by final state 



Figure 2: Left: Electron Energy from $v$-e process; Right: Flux constraint. [2]
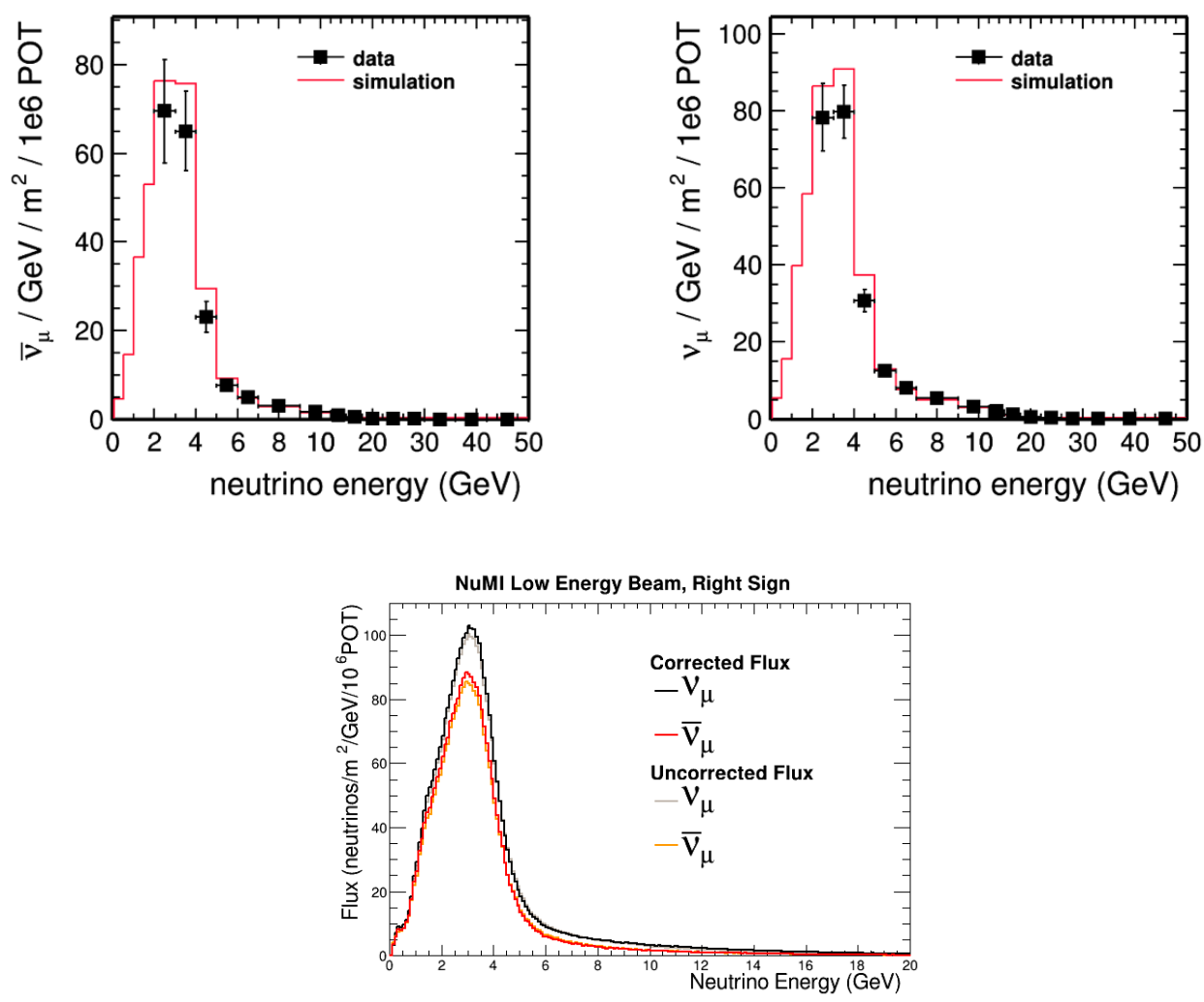

Figure 3: Flux prediction from Low-Nu method for neutrinos (top left) and antineutrinos (top right). Flux prediction for neutrino and antineutrino corrected and uncorrected usign the Low-Nu method (bottom). [3] 



Figure 4: CC Inclusives Cross Section and ratios [4].

interactions (FSI). The distributions in kinematics variables for both samples are based on muon track reconstruction. The measured cross section shape of charged current pion production versus pions kinematics seem to be independent of the FSI used in the generators
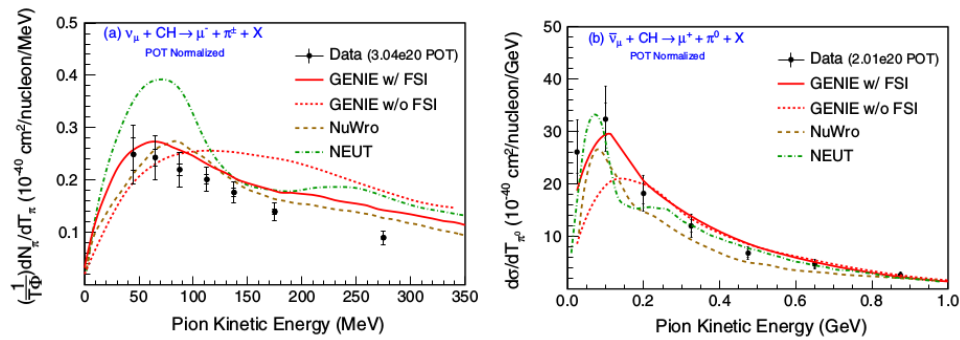

Figure 5: Shape of cross section versus pions kinematics. Kinectic energy for charged (left) and neutral (right) $\pi$ 's. [5]

\section{7. $v_{\mu}$ Charged Current Single $\pi^{0}$ Production}

MINERvA has studied the CC $\pi^{0}$ production. This analysis considers that the $\pi^{0}$ comes either from a $\Delta^{+}(1232)$ resonance or is generated by Deep Inelastic Scattering (DIS). The plots in Figure 6 show the measured cross section as function of the 4-momentum transfered, pion kinetic energy and pion angle compared to GENIE and NuWro predictions. The measured cross sections, mainly as function of the kinetic energy, provide a detailed view of this pion production and give information for pion FSI model constraints. The $\Delta$ resonance decay angle has been measured for the first time (GENIE and NuWro assume the that the $\Delta$ decays isotropically).

\section{Diffractive Neutral Pion Production}

MINERvA has used the observed data excess in $\mathrm{dE} / \mathrm{dx}$ distribution in the photon region to study the diffractive $\pi^{0}$ production. Originality, this distribution was done to help in the selection of the electron-neutrino ccqe. The excess is signaled by the presence of 2 electromagnetic showers, that are supposed to come from the $\pi^{0}$ decay, by the presence of a proton upstream of the showers and by no other energy. Plots in Figure 7 show how the data excess shape compares to NC $\pi^{0}$ and 

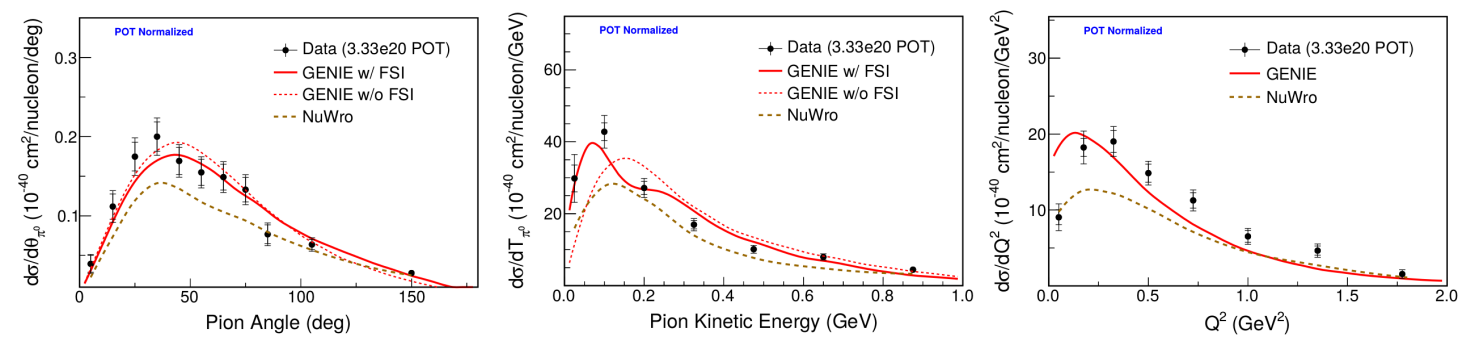

Figure 6: $\pi^{0}$ cross section as function of $\pi$ angle, kinectic energy and $Q^{2}$. [6].

coherent $\pi^{0}$ as function of reconstructed energy and of $E \theta^{2}$. This is the first direct experimental observation and characterization of this process, that is not included in the generator.
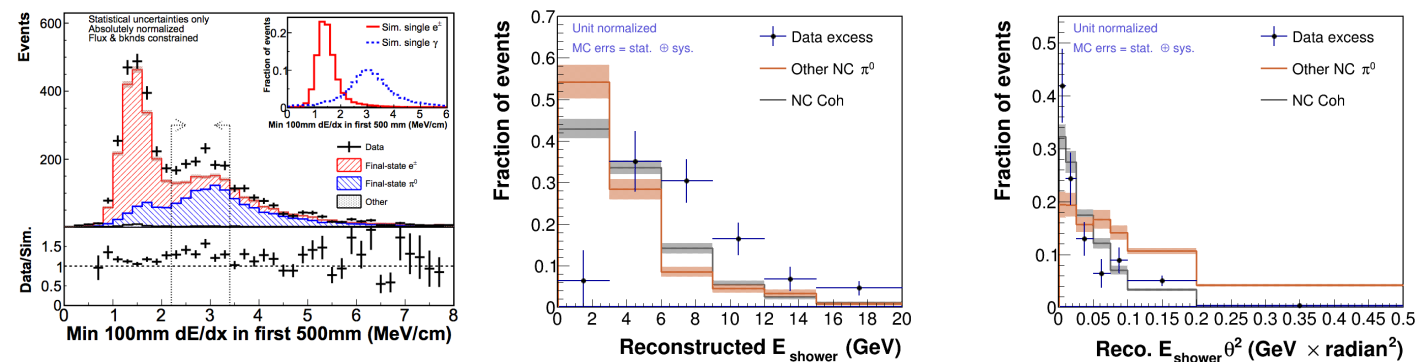

Figure 7: Data excess on $\mathrm{dE} / \mathrm{dx}$ photon region (left); as a function of the electron energy (center); as a function of $\mathrm{E} \theta^{2}[7]$ (right).

\section{Charged Current $\mathrm{K}^{+}$Production}

Timing information is used to isolate about 900 event candidates. This is the first high statistic measurement of $\mathrm{K}^{+}$production in neutrino CC interactions [8], and provides additional constraints to the Final State Interactions. Left plot of Figure 8 shows that Kaon kinetic energy agrees better with GENIE than with NuWro what tells us about the importance of improving the low-W DIS model for $\mathrm{K}^{+}$production.

\section{Coherent $\mathrm{K}^{+}$Production}

Coherent $\mathrm{K}^{+}$production is a very rare inelastic electroweak process. The kinematics of the final state is used to reconstruct the small momentum transferred to the nucleus. Kaon candidate events are identified via cuts on the vertex energy, cuts on $t$, and by scanning the event displays to remove $\pi^{0}$ 's. A total of 6 candidate events where found in the signal region [9].

\section{Neutral Current $\mathrm{K}^{+}$Production}

MINERvA has recently published results of Neutral Current Kaon production [10]. The $K^{+}$ production by atmospheric neutrinos is a background in the proton decay process. Particularly 
problematic in Cherenkov detectors, where $\mathrm{NC} \mathrm{K}^{+}$event with no particles above Cherenkov threshold will fake the signal process. The mismodeling rates for $K^{+}$is a problem in liquid argon detectors.
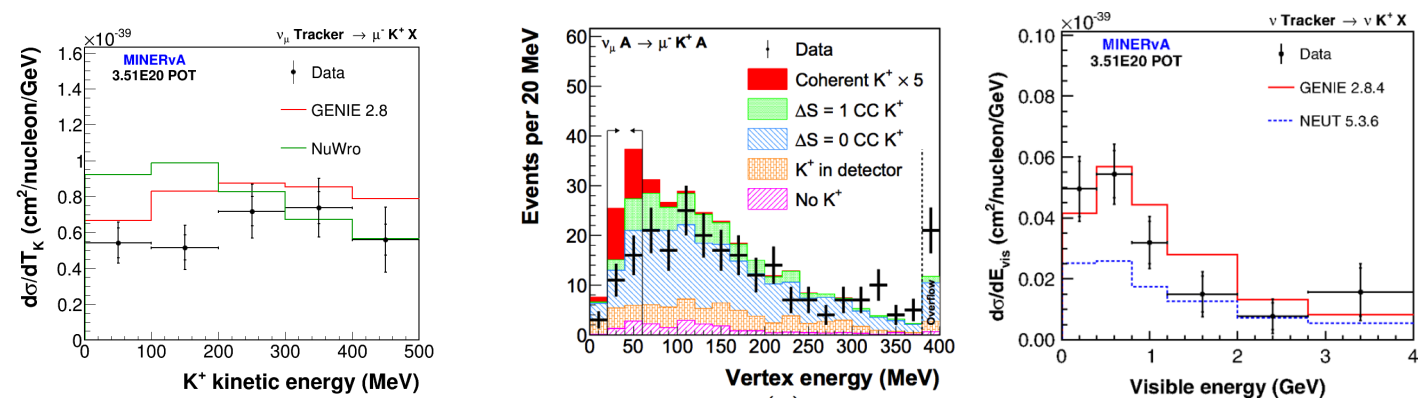

Figure 8: Netrino $K^{+}$production: Kinetic Energy for $\mathrm{CC} K^{+}$[8] (left); vertex energy for Coherent $K^{+}$[9] (center); visible energy for NC $K^{+}$[10] (right).

\section{Status and Future Plans}

MINERvA is taking antineutrino data in the medium energy beam configuration. The neutrino mode ended on February 202017 after collecting a final sample with almost 12 E20 POT. Current antineutrino run is expected to continue until, at least, 2019. MINERvA is working in analysis of quasi-eleastic, pion production, DIS and inclusive studies on iron, carbon and lead. A $10 \%$ reduction of systematics is expected and MINERvA will have more statistics for the neutrinoelectron scattering used for the flux Constraint. The future Medium Energy results will increase the sensitivity of nuclear effects studies.

\section{Conclusions}

The MINEvA Experiment has already published 22 papers. Neutrino cross section studies are important for neutrino oscillation measurements and for the understanding of the nature of neutrino-nucleus scattering. MINERvA has done precise studies of neutrino interaction in the 1$20 \mathrm{GeV}$ range improving our knowledge of the flux, neutrino charged current interactions, and nuclear dependence of neutrino interactions. MINERvA is setting constraints for many channels. MINERvA studies of kaons provide the first direct data constraint for background from atmospheric neutrinos. MINERvA results are helping to resolve some historical discrepancies between experiments and are important for minimizing systematic errors in present and future oscillation experiments.

\section{References}

[1] ”Neutrino Flux Predictions for the NuMI Beam, Phys. Rev. D 94, 092005 (2016)

[2] Measurement of Neutrino Flux using Neutrino-Electron Elastic Scattering, Phys. Rev. D 93, 112007 (2016). 
[3] "Measurements of the Inclusive Neutrino and Antineutrino Charged Current Cross Sections in MINERvA Using the Low- $v$ Flux Method", Phys. Rev. D 94, 112007 (2016).

[4] "Measurement of the antineutrino to neutrino charged-current interaction cross section ratio on carbon”, Phys. Rev. D 95, 072009 (2017).

[5] "Cross sections for neutrino and antineutrino induced pion production on hydrocarbon in the few $\mathrm{GeV}$ region using MINERvA”, Phys. Rev. D 94, 052005 (2016).

[6] "Measurement of $v_{\mu}$ charged-current single $\pi^{0}$ production on hydrocarbon in the few-GeV region using MINERvA”, Phys. Rev. D 96, 072003 (2017).

[7] "Evidence for diffractive neutral pion production from hydrogen in Neutrino Interactions on hydrocarbon", Phys. Rev. Lett. 117, 111801 (2016).

[8] "Measurement of $K^{+}$production in charged-current $v_{\mu}$ interactions", Phys. Rev. D 94012002 (2016).

[9] "First evidence of coherent $K^{+}$meson production in neutrino-nucleus scattering", Phys. Rev. Lett. 117, 061802 (2016).

[10] "Measurement of neutral-current $K^{+}$production by neutrinos using MINERvA", Phys. Rev. Lett. 199, 0011802 (2017). 